\title{
Autoimmune Diabetes in the NOD Mouse: An Essential Role of Fas-FasL Signaling in $\beta$ Cell Apoptosis
}

\author{
DIEGO G. SILVA,${ }^{a, b}$ LUIS SOCHA,${ }^{a, b}$ BRETT CHARLTON,${ }^{b}$ WILLIAM COWDEN,${ }^{b}$ \\ AND NIKOLAI PETROVSKY $Y^{a, b}$ \\ ${ }^{a}$ Autoimmunity Research Unit, The Canberra Hospital, Canberra, Australia \\ ${ }^{b}$ John Curtin School of Medical Research, Canberra, Australia
}

\begin{abstract}
Despite evidence that both Fas and FasL can be expressed in pancreatic islets, there has been considerable controversy regarding the potential role of Fas signaling in autoimmune $\beta$ cell death. Using the HIPFasL model, we have been able to demonstrate that, in the presence of an inflammatory infiltrate, FasL-expressing $\beta$ cells are exquisitely sensitive to Fas-mediated apoptosis and that this can be blocked by preventing FasL-Fas interaction. This points to a highly important role of Fas-FasL interaction in autoimmune $\beta$ cell death.
\end{abstract}

KEYWORDS: Fas ligand; autoimmunity; type 1 diabetes; NOD; apoptosis

\section{INTRODUCTION}

Autoimmune diabetes of the NOD mouse is characterized by destruction of pancreatic $\beta$ cells by a process of programmed cell death, also called apoptosis. ${ }^{1}$ Multiple signaling pathways can lead to apoptosis, mainly working through activation of cell surface molecules such as the TNF and Fas receptors, both of which belong to the TNF receptor superfamily. Binding of Fas (CD95) to its ligand (FasL) triggers a series of downstream events including activation of the caspase pathway and this ultimately leads to DNA fragmentation and apoptotic cell death. Despite evidence, as detailed below, that both Fas and FasL can be expressed in pancreatic islets, there has been considerable controversy regarding the potential role of Fas signaling in autoimmune $\beta$ cell death. Fas is expressed by mouse and human $\beta$ cells after stimulation with inflammatory cytokines (e.g., IFN- $\gamma$, IL-1, and/or TNF- $\alpha$ ) ${ }^{2-4}$ FasL is also present on islet cells ${ }^{5,6}$ and, by dual labeling studies, has been shown to be expressed by $\beta$ cells of diabetic NOD mice and nondiabetic C57BL/6 mice. ${ }^{7}$ FasL is also expressed by islet-infiltrating inflammatory cells and by islet $\alpha$ cells. $^{5}$

Address for correspondence: Professor Nikolai Petrovsky, Head, Autoimmunity Research Unit, The Canberra Hospital, P.O. Box 11, Woden ACT 2606, Australia. Voice: +612-62442595; fax: +612-62603372.

nikolai.petrovsky@anu.edu.au

Ann. N.Y. Acad. Sci. 1005: 161-165 (2003). (C) 2003 New York Academy of Sciences. doi: 10.1196/annals.1288.018 


\section{HIPFASL TRANSGENIC MICE}

In confirmation of the importance of Fas-FasL interaction in the development of autoimmune diabetes, NOD mice where FasL is transgenically expressed on the $\beta$ cell surface (HIPFasL mice) demonstrate pronounced $\beta$ cell destruction and accelerated diabetes. $^{2,8 a}$ HIPFasL mice demonstrate a mononuclear cell islet infiltrate with associated $\beta$ cell destruction, similar to the picture seen in spontaneous diabetes of the NOD mouse. ${ }^{8 b}$ Diabetes was not seen when the HIPFasL transgene was crossed onto various nondiabetes-prone strains including C57BL/6 mice, indicating that the NOD background was necessary for the FasL transgene to mediate diabetes. ${ }^{8 \mathrm{~b}}$ Similarly, HIPFasL mice with the SCID mutation, which have no T cells, were also protected against diabetes. The above findings suggest that the FasL transgene only mediates $\beta$ cell death in the context of an inflammatory cell islet infiltrate such as occurs in all NOD mice even in the absence of overt diabetes.

\section{SUSCEPTIBILITY OF HIPFASL ISLETS TO IMMUNE DESTRUCTION IN VIVO}

From the above results, we hypothesized that HIPFasL $\beta$ cells have an increased susceptibility to apoptosis, but only in the event of exposure of HIPFasL $\beta$ cells to an inflammatory environment capable of upregulating $\beta$ cell Fas expression. Coexpression of Fas and FasL would then rapidly lead to $\beta$ cell suicide or fratricide. This would explain why HIPFasLSCID mice, which have no insulitis or T cell-derived cytokines, do not develop diabetes. ${ }^{8 \mathrm{~b}}$ We decided, therefore, to test the relative susceptibility of HIPFasL and nontransgenic NOD islets to destruction when exposed to a mononuclear infiltrate after grafting into nondiabetic NOD mice. HIPFasL and nontransgenic NOD islets were grafted at either end of the kidney capsule of 70-dayold NOD mice. The kidneys were then removed 60 days later and the degree of preservation or destruction of the HIPFasL and nontransgenic NOD grafts compared. Macroscopically, NOD grafts were vascularized and showed a normal graft structure, whereas the HIPFasL grafts showed all the features of destructive insulitis with the graft site being swollen and the normal graft morphology lost (FIGS. 1a and 1c). While hematoxylin-and-eosin and insulin staining revealed mononuclear cellular infiltrate in both HIPFasL and NOD islet grafts, the HIPFasL islet grafts had evidence of widespread destruction with an intra-islet infiltrate and loss of islet architecture. By comparison, nontransgenic NOD grafts revealed minimal islet destruction (FIGS. 1b and 1d).

\section{SUSCEPTIBILITY OF HIPFASL/NOD ISLETS TO CYTOKINE-INDUCED APOPTOSIS}

The question then arose as to whether the cellular infiltrate in the islets was necessary for FasL-mediated $\beta$ cell death or, alternatively, whether it is simply the cytokines produced by this infiltrate that were necessary. As Fas expression is known to be induced on $\beta$ cells by incubation in IFN- $\gamma$ and IL-1, ${ }^{9}$ we next asked whether HIPFasL $\beta$ cells were more susceptible to cytokine-induced apoptosis than NOD 
NOD islets graft

a)

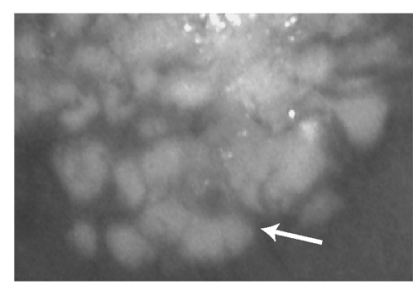

HIPFasL islets graft

c)

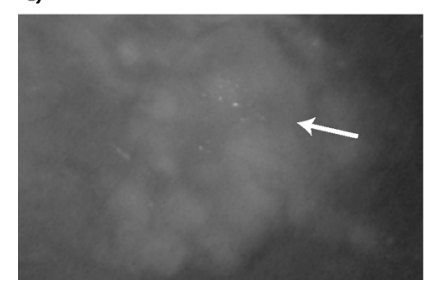

b)

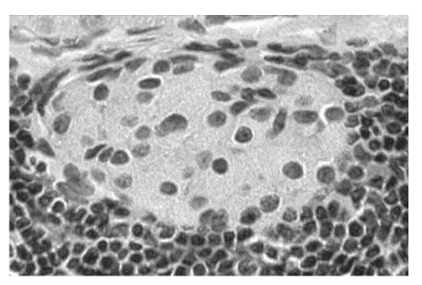

d)

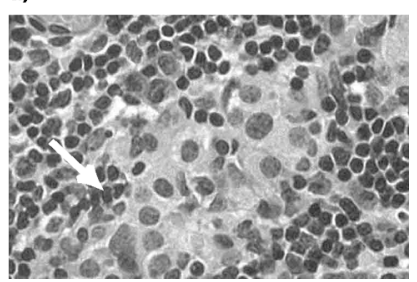

FIGURE 1. HIPFasL and NOD islets were cotransplanted under the kidney capsule of 70-day-old NOD mice and harvested 60 days later. Grafts from NOD donors (a, b) were clearly identified, with macroscopic (a) and microscopic (b) characteristics of nondestructive lesions. In contrast, HIPFasL islet grafts $(\mathbf{c}, \mathbf{d})$ showed signs of destructive lesions: grafts were cloudy and with no well-defined islets (c) and showed intra-islet infiltrate with islet destruction (d). The figure shows one representative animal.

$\beta$ cell islets and, if so, whether this increased apoptosis could be blocked by FasLneutralizing antibody. Islets were isolated from HIPFasL mice and nontransgenic NOD controls, and cultured at 30 islets/well for $24 \mathrm{~h}$ in the presence or absence of IL- $1 \beta$ and IFN- $\gamma$. The percentage of apoptotic HIPFasL $\beta$ cells after $24 \mathrm{~h}$ of cytokine stimulation was approximately three times that of nontransgenic NOD $\beta$ cells, indicating that $\beta$ cells from HIPFasL-positive mice are indeed highly susceptible to cytokine-induced apoptosis compared to nontransgenic NOD $\beta$ cells. ${ }^{8 b}$ Furthermore, the increased cytokine-induced apoptosis of HIPFasL $\beta$ cells was prevented by the addition of neutralizing antibody to FasL (MFL3 clone), confirming that Fas-FasL interaction did underlie the observed increase of $\beta$ cell apoptosis. ${ }^{8 b}$

\section{DISCUSSION}

In autoimmune diabetes, islet $\beta$ cells are chronically exposed to a milieu of inflammatory cytokines. In the NOD mouse, mononuclear invasion of the islets starts soon after weaning. The presence of this inflammatory environment markedly affects normal $\beta$ cell biology, with increased or reduced expression of over 150 genes involved in a whole range of cellular processes, ${ }^{10}$ including upregulation of cell surface receptors such as Fas. The presence of an inflammatory infiltrate is essential 
during the development of autoimmune diabetes as neither NOD nor HIPFasL mice carrying the SCID mutation develop diabetes. ${ }^{8 \mathrm{~b}}$

Until recently, the mechanism by which the inflammatory infiltrate mediated $\beta$ cell apoptosis remained uncertain, with Fas, ${ }^{9,11}$ nitric oxide, ${ }^{3,12,13}$ free radical stress, ${ }^{14,15}$ and perforin ${ }^{16-18}$ all being postulated to play a role. Studies by ourselves ${ }^{8}$ and others ${ }^{19,20}$ have increasingly emphasized the importance of Fas-mediated $\beta$ cell apoptosis in NOD autoimmune diabetes. Using the HIPFasL model, we have been able to demonstrate that, in the presence of an inflammatory infiltrate, FasL-expressing $\beta$ cells are exquisitely sensitive to Fas-mediated apoptosis and that this can be blocked by preventing FasL-Fas interaction with FasL-neutralizing antibody. Also, the effect of this inflammatory infiltrate can be reproduced with the cytokines, IFN- $\gamma$ and IL-1, which together upregulate $\beta$ cell Fas expression. Together, this evidence points to a highly important role of Fas-FasL interaction in autoimmune $\beta$ cell death. Clearly, like so many other body systems, there is likely to be some redundancy built into the system such that other $\beta$ cell death pathways are also likely to be relevant to autoimmune diabetes, albeit to a lesser degree than Fas. However, it does stress the importance of interrupting the Fas signaling pathway if autoimmune $\beta$ cell death is to be prevented. We are now interested in looking at potential synergistic effects of blocking multiple apoptosis pathways including Fas in combination to see whether this is even more effective in preventing $\beta$ cell death than blocking Fas alone.

\section{ACKNOWLEDGMENTS}

This work was supported by the Canberra Hospital Salaried Specialists Private Practice Fund.

\section{REFERENCES}

1. Kurrer, M.O., S.V. Pakala, H.L. Hanson \& J.D. Katz. 1997. Beta cell apoptosis in T cell-mediated autoimmune diabetes. Proc. Natl. Acad. Sci. USA 94: 213.

2. Chervonsky, A.V., Y. WANG, F.S. Wong et al. 1997. The role of Fas in autoimmune diabetes. Cell 89: 17.

3. Stassi, G., R. De Maria, G. Trucco et al. 1997. Nitric oxide primes pancreatic beta cells for Fas-mediated destruction in insulin-dependent diabetes mellitus. J. Exp. Med. 186: 1193.

4. Suarez-Pinzon, W., O. Sorensen, R.C. Bleackley et al. 1999. Beta-cell destruction in NOD mice correlates with Fas (CD95) expression on beta-cells and proinflammatory cytokine expression in islets. Diabetes 48: 21.

5. Signore, A., A. AnNovazZI, E. Procaccini et al. 1997. CD95 and CD95-ligand expression in endocrine pancreas of NOD, NOR, and BALB/c mice. Diabetologia 40: 1476.

6. Loweth, A.C., G.T. Williams, R.F. James et al. 1998. Human islets of Langerhans express Fas ligand and undergo apoptosis in response to interleukin-1beta and Fas ligation. Diabetes 47: 727.

7. REDD, S., S. GINN \& J.M. Ross. 2002. Fas and Fas ligand immunolocalization in pancreatic islets of NOD mice during spontaneous and cyclophosphamide-accelerated diabetes. Histochem. J. 34: 1.

8. (a) Petrovsky, N., D. Silva, L. Socha et al. 2002. The role of Fas ligand in beta cell destruction in autoimmune diabetes of NOD mice. Ann. N.Y. Acad. Sci. 958: 204; 
(b) Silva, D., N. Petrovsky, L. Socha et al. 2003. Mechanisms of accelerated immune-mediated diabetes resulting from islet beta-cell expression of a FAS ligand transgene. J. Immunol. 170: 4996.

9. Thomas, H.E., R. Darwiche, J.A. Corbett \& T.W. Kay. 1999. Evidence that beta cell death in the nonobese diabetic mouse is Fas independent. J. Immunol. 163: 1562.

10. Cardozo, A.K., M. Kruhoffer, R. Leeman et al. 2001. Identification of novel cytokine-induced genes in pancreatic beta-cells by high-density oligonucleotide arrays. Diabetes 50: 909.

11. Su, X., Q. Hu, J.M. Kristan et al. 2000. Significant role for Fas in the pathogenesis of autoimmune diabetes. J. Immunol. 164: 2523.

12. Thomas, H.E. \& T.W. KaY. 2000. Beta cell destruction in the development of autoimmune diabetes in the non-obese diabetic (NOD) mouse. Diabetes Metab. Res. Rev. 16: 251 .

13. Zumsteg, U., S. Frigerio \& G.A. Hollander. 2000. Nitric oxide production and Fas surface expression mediate two independent pathways of cytokine-induced murine beta-cell damage. Diabetes 49: 39 .

14. WeSt, I.C. 2000. Radicals and oxidative stress in diabetes. Diabetes Med. 17: 171.

15. Suarez-Pinzon, W.L., C. Szabo \& A. Rabinovitch. 1997. Development of autoimmune diabetes in NOD mice is associated with the formation of peroxynitrite in pancreatic islet beta-cells. Diabetes 46: 907.

16. Kay, T.W., H.E. Thomas, L.C. Harrison \& J. Allison. 2000. The beta cell in autoimmune diabetes: many mechanisms and pathways of loss. Trends Endocrinol. Metab. 11: 11.

17. Yoon, J.W. \& H.S. JUN. 2001. Cellular and molecular pathogenic mechanisms of insulin-dependent diabetes mellitus. Ann. N.Y. Acad. Sci. 928: 200.

18. Kreuwel, H.T. \& L.A. Sherman. 2001. The role of Fas-FasL in CD8+ T-cell-mediated insulin-dependent diabetes mellitus (IDDM). J. Clin. Immunol. 21: 15.

19. Mahiou, J., U. Walter, F. Lepault et al. 2001. In vivo blockade of the Fas-Fas ligand pathway inhibits cyclophosphamide-induced diabetes in NOD mice. J. Autoimmun. 16: 431 .

20. Suarez-Pinzon, W.L., R.F. Power \& A. Rabinovitch. 2000. Fas ligand-mediated mechanisms are involved in autoimmune destruction of islet beta cells in non-obese diabetic mice. Diabetologia 43: 1149 . 